\title{
Generally covariant geometric momentum, gauge potential and a Dirac fermion on a two-dimensional sphere
}

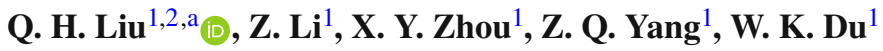 \\ ${ }^{1}$ School for Theoretical Physics, College of Physics and Electronics, Hunan University, Changsha 410082, China \\ ${ }^{2}$ Synergetic Innovation Center for Quantum Effects and Applications (SICQEA), Hunan Normal University, Changsha 410081, China
}

Received: 2 July 2019 / Accepted: 17 August 2019 / Published online: 24 August 2019

(C) The Author(s) 2019

\begin{abstract}
For a particle that is constrained on an $(N-1)$ dimensional $(N \geq 2)$ curved surface $\Sigma^{N-1}$, the Cartesian components of its momentum in $N$-dimensional flat space are believed to offer a proper form of momentum for the particle on the surface, which is called the geometric momentum as it depends on the mean curvature. Once the momentum becomes generally covariant as to be applicable to spin particles on the surface, the spin connection part in it can be interpreted as a gauge potential. The principal findings are twofold. The first is a general framework of quantum conditions for a spin particle on the hypersurface $\Sigma^{N-1}$, and the generalized angular momentum is defined on hypersphere $S^{N-1}$ as one consequence of the generally covariant geometric momentum. The second is devoted to a study of a Dirac fermion on a two-dimensional sphere and we show that there is the generalized angular momentum whose three cartesian components form the $s u(2)$ algebra, demonstrated to be of geometric origin but obtained before by consideration of dynamics of the particle. Moreover, we show that there is no curvature-induced geometric potential for the spin half particle.
\end{abstract}

\section{Introduction}

In quantum mechanics, there are fundamental quantum conditions (FQCs) $\left[x_{i}, x_{j}\right]=0,\left[x_{i}, p_{j}\right]=i \hbar \delta_{i j}$, and $\left[p_{i}, p_{j}\right]=0$, which are defined by the commutation relations between positions $x_{i}$ and momenta $p_{i}(i, j, k, l=$ $1,2,3, \ldots, N$ ) where $N$ denotes the number of dimensions of the flat space in which the particle moves [1]. In position representation, the momentum operator takes simple form as $\mathbf{p}=-i \hbar \nabla$ where $\nabla \equiv \mathbf{e}_{i} \partial / \partial x_{i}$ is the ordinary gradient operator, and $N$ mutually orthogonal unit vectors $\mathbf{e}_{i}$ span the $N$ dimensional Euclidean space $E^{N}$. Hereafter the Einstein

a e-mail: quanhuiliu@gmail.com summation convention over repeated indices is used. When the particle is constrained to remain on a hypersurface $\Sigma^{N-1}$ embedded in $E^{N}$, in classical mechanics, we can define Dirac bracket $\{A, B\}_{D}$ instead of the usual Poisson one $\{A, B\}$ for the two functions $A$ and $B$ of both $x_{i}$ and $p_{i}$ [2-6],

$$
\{A, B\}_{D} \equiv\{A, B\}-\left\{A, \varphi_{\alpha}\right\} C_{\alpha \beta}^{-1}\left\{\varphi_{\beta}, B\right\},
$$

where $\varphi_{\alpha}=0(\alpha=1,2)$ are two constraint conditions, and $\varphi_{1} \equiv f(\mathbf{x})(=0)$ is the equation of surface, and $\varphi_{2} \equiv$ $\mathbf{p} \cdot \nabla f(\mathbf{x})(=0)$ shows the motion to be tangential to the surface, and the matrix elements $C_{\alpha \beta}$ is defined by $C_{\alpha \beta}=$ $\left\{\varphi_{\alpha}, \varphi_{\beta}\right\}$. The equation of surface $f(\mathbf{x})=0$ can be so chosen that $|\nabla f(\mathbf{x})|=1$ thus $\mathbf{n} \equiv \nabla f(\mathbf{x})=\mathbf{e}_{i} n_{i}$ is the normal vector at a local point $u^{\mu}(\mu, v, \alpha, \beta=1,2,3, \ldots, N-1)$ on the surface. The relation between positions $\mathbf{x}$ in $E^{N}$ and the local coordinates $u^{\mu}$ on $\Sigma^{N-1}$ is $\mathbf{x}=\mathbf{x}\left(u^{\mu}\right)$, and $\mathbf{x}^{\mu}=\partial \mathbf{x} / \partial u_{\mu}$ is the $\mu$ th contravariant component of the natural frame on the point. The usual canonical commutation relations are hypothesized to be given by $[A, B]=i \hbar\left\{[A, B]_{D}\right\}_{\text {Hermitian }}$ [2] where $O_{\text {Hermitian }}$ stands for a Hermitian operator of an observable $O$. Explicitly, Dirac brackets for variables $x_{i}$ and $p_{i}$ are given by $\left[x_{i}, x_{j}\right]_{D}=0,\left[x_{i}, p_{j}\right]_{D}=\left(\delta_{i j}-n_{i} n_{j}\right)$, and $\left[p_{i}, p_{j}\right]_{D}=-\left(n_{i} n_{k, j}-n_{j} n_{k, i}\right) p_{k}$. The FQCs [2] are thus [3-6],

$$
\begin{aligned}
{\left[x_{i}, x_{j}\right] } & =0, \quad\left[x_{i}, p_{j}\right]=i \hbar\left(\delta_{i j}-n_{i} n_{j}\right), \text { and } \\
{\left[p_{i}, p_{j}\right] } & =-i \hbar\left\{\left(n_{i} n_{k, j}-n_{j} n_{k, i}\right) p_{k}\right\}_{\text {Hermitian }},
\end{aligned}
$$

This set of the FQCs (2) is highly non-trivial, from which it is in general impossible to uniquely construct the momenta $p_{i}$. Our propose of the proper form of the momentum for a spinless particle was [7-11],

$\mathbf{p}=-i \hbar\left(\nabla_{\Sigma}+M \mathbf{n} / 2\right)$,

where $\nabla_{\Sigma} \equiv \mathbf{e}_{i}\left(\delta_{i j}-n_{i} n_{j}\right) \partial_{j}=\nabla-\mathbf{n} \partial_{n}=\mathbf{x}^{\mu} \partial_{\mu}$ is the gradient operator, and the mean curvature $M \equiv-\nabla_{\Sigma} \cdot \mathbf{n}$ is defined 
by the sum of the all principal curvatures. Since the mean curvature $M$ is an extrinsic curvature, this form of momentum (3) is fundamentally different from the canonical ones in curvilinear coordinates for (3) depends on the geometric invariants, which can then be conveniently called as geometric momentum [7-13]. This momentum satisfies following simplest form of $\left[p_{i}, p_{j}\right][11]$,

$$
\begin{aligned}
{\left[p_{i}, p_{j}\right]=} & -i \hbar\left\{\left(n_{i} n_{k, j}-n_{j} n_{k, i}\right) p_{k}\right. \\
& \left.+p_{k}\left(n_{i} n_{k, j}-n_{j} n_{k, i}\right)\right\} / 2 .
\end{aligned}
$$

This momentum (3) can be obtained by many different ways including: the hermiticity requirement on derivative operator $-i \hbar \nabla_{\Sigma}$ [7], and compatibility of constraint condition $\mathbf{n} \cdot \mathbf{p}+\mathbf{p} \cdot \mathbf{n}=0$ which means in quantum mechanics that the motion is perpendicular to the surface normal vector $\mathbf{n}[8,9]$, and thin-layer quantization or confining potential formalism which instead considers that particle is confined onto the surface $\Sigma^{N-1}$ by means of introduction of a confinement potential along the normal direction of the surface [10], and dynamical quantum conditions (DQCs) [11] (see also (15)-(17) in the Sect. 4), etc. $[4,13,14]$ Experimental justification was performed by comparison of the interference spots formed by the surface plasmon polariton propagating on a cylindrical surface, predicted by the introduction of the geometric momentum or not [12], respectively. Some of previous discussions deal with quite general case in higher dimensions $[4,9,11]$, some of them $[7,8,10,13]$ are mainly for a particle on $\Sigma^{2}$.

The geometric momentum (3) suffices to act on a quantum state of single component. However, quantum states on the surfaces are usually multi-component such as spinors [1520], requiring that the momentum to be generally covariant. In fact, the generally covariant geometric momentum (GCGM) is at hand, though not yet explicitly written before. The present paper shows that the GCGM is a useful and convenient physical quantity.

This paper is mainly divided into two parts. Sections $2-4$ are devoted to build up a general formulation between the GCGM and quantization conditions. Section 5 and 6 study the Dirac fermion on $S^{2}$. In Sect. 2, the introduction of the GCGM is made and its dependence on the gauge potential is transparent. In Sect. 3, though we do not know in general whether the GCGM satisfies the quantization condition (4), or satisfies other forms of the last one of the FQCs (2) $\left[p_{i}, p_{j}\right]=-i \hbar\left\{\left(n_{i} n_{k, j}-n_{j} n_{k, i}\right) p_{k}\right\}_{\text {Hermitian }}$, the FQCs $\left[p_{i}, p_{j}\right]$ for a Dirac fermion on $S^{N-1}$ have a well-defined consequence to define a generalized angular momentum. In Sect. 4, we show how the self-consistent consideration of the quantization conditions leads us to the DQCs for a relativistic particle on $\Sigma^{N-1}$. In Sect. 5, we deal with the Dirac fermion on $S^{2}$, and use FQCs and GCGM to reproduce the same generalized angular momentum obtained before by means of a purely dynamical consideration. In Sect. 6, we use DQCs and GCGM to check whether the curvature-induced geometric potential exists for a Dirac fermion on $S^{2}$, and results show that no such a potential. Final Sect. 7 is a brief conclusion.

\section{Generally covariant geometric momentum and gauge potential}

This section is to show that the GCGM is at the ready, and its dependence on the gauge potential is transparent.

To note $\nabla_{\Sigma} \equiv \mathbf{e}_{i}\left(\delta_{i j}-n_{i} n_{j}\right) \partial_{j}=\mathbf{r}^{\mu} \partial_{\mu}$, and the usual derivative $\partial_{\mu}\left(\equiv \partial / \partial u^{\mu}\right)$ in (3) can be made generally covariant by a simple replacement [15-20],

$\partial_{\mu} \longrightarrow D_{\mu} \equiv \partial_{\mu}+i \Omega_{\mu}$

and we immediately have,

$$
\begin{aligned}
\mathbf{p} & =-i \hbar\left(\nabla_{\Sigma}+\frac{M \mathbf{n}}{2}+i \mathbf{r}^{\mu} \Omega_{\mu}\right) \\
& =-i \hbar\left(\nabla_{\Sigma}+\frac{M \mathbf{n}}{2}\right)+\hbar \mathbf{r}^{\mu} \Omega_{\mu},
\end{aligned}
$$

where $\Omega_{\mu}=(-i / 8) \omega_{\mu}^{a b}\left[\gamma_{a}, \gamma_{b}\right]$ in which $\omega_{\mu}^{a b}$ are the spin connections [16-22] and $\gamma_{a}(a, b=0,1,2, \ldots, N)$ are Dirac spin matrices. In comparison of (6) with the usual kinematical momentum $\mathbf{p}=-i \hbar \nabla-q \mathbf{A}$ in presence of magnetic potential $\mathbf{A}$, we see that there is an equivalent magnetic potential $\mathbf{A}$ that can be defined by $q \mathbf{A} \equiv-\hbar \mathbf{r}^{\mu} \Omega_{\mu}$, in which the charge $q$ can be understood as an effective interaction strength between the charge and the field. Once writing $\Omega_{\mu}$ as a product of $\omega_{\mu}^{a b} / 4$ and $Q_{a b} \equiv\left[\gamma_{a}, \gamma_{b}\right] /(2 i)$, we can take the eigenvalues of the matrices $Q_{a b}$ as an effective interaction strength [21,22]. This form of GCGM (6) is applicable to particles, relativistically or not, massively or not.

Two observations concerning the GCGM are in following.

1. Once the surface $\Sigma^{N-1}$ is embedded into higher flat space in $E^{N+p}(N \succeq 2)$ with a positive integer $p \succeq 1$, we have another way of making the derivative $\partial_{\mu}$ in (3) generally covariant by replacement [21-32],

$\partial_{\mu} \longrightarrow D_{\mu} \equiv \partial_{\mu}+i W_{\mu}$

and we have as well,

$$
\begin{aligned}
\mathbf{p} & =-i \hbar\left(\nabla_{\Sigma}+\frac{M \mathbf{n}}{2}+i \mathbf{r}^{\mu} W_{\mu}\right) \\
& =-i \hbar\left(\nabla_{\Sigma}+\frac{M \mathbf{n}}{2}\right)+\hbar \mathbf{r}^{\mu} W_{\mu},
\end{aligned}
$$

where $\hbar W_{\mu}=N_{\mu}^{A B} L_{A B} / 2$ in which $N_{\mu}^{A B}(A, B=$ $N, N+1, \ldots, N+p)$ stand for the normal connections 
determined by the so-called normal fundamental form, and $L_{A B}$ are angular momentum in the normal space. It was realized that the normal connections $N_{\mu}^{A B} / 2$ and spin connections $\omega_{\mu}^{a b} / 4$ can take identical form for $S^{N-1}$, which was used to explore a possibly geometrical origin of spin in contrast to that is generally accepted to be connected with relativity [33].

2. Starting from replacement (5) or (7), we can define the gauge potential $A_{\mu} \equiv-\hbar \Omega_{\mu}$, or $A_{\mu} \equiv-\hbar W_{\mu}$. Therefore the field strength $F_{\mu \nu}$ can be defined by [21$32,34,35]$,

$$
F_{\mu \nu} \equiv \partial_{\mu} A_{\nu}-\partial_{\nu} A_{\mu}-i\left[A_{\mu}, A_{\nu}\right]=i\left[D_{\mu}, D_{\nu}\right] .
$$

Whether this gauge field $F_{\mu \nu}$ is abelian or non-abelian depends on whether the commutators $\left[A_{\mu}, A_{\nu}\right]$ vanish or not. In terms of the GCGM, we have the gauge potential in Cartesian coordinates,

$$
\mathbf{A} \equiv \mathbf{r}^{\mu} A_{\mu}=-\hbar \mathbf{r}^{\mu} \Omega_{\mu}, \quad \text { or }-\hbar \mathbf{r}^{\mu} W_{\mu}
$$

Now the introduction of the GCGM is complete. However, whether it satisfies the quantization conditions (4), or satisfies other forms of the last ones of the FQCs (2) $\left[p_{i}, p_{j}\right]=-i \hbar\left\{\left(n_{i} n_{k, j}-n_{j} n_{k, i}\right) p_{k}\right\}_{\text {Hermitian }}$, is not so easily resolved in general. We leave it as an open problem though we believe it is true. For the special case of a Dirac fermion on $S^{N-1}$, quantum conditions $\left[p_{i}, p_{j}\right]$ are welldefined and lead to a generalized angular momentum, which will be discussed in next section.

\section{Fundamental quantum conditions $\left[p_{i}, p_{j}\right]$ for a Dirac fermion on $S^{N-1}$}

The hypersphere $S^{N-1}$ of radius $R$ in $N$-dimensional flat space $E^{N}$ can be,

$f(\mathbf{x}) \equiv \frac{1}{2 R}\left(\sum_{i=1}^{N} x_{i}^{2}-R^{2}\right)=0$.

The fundamental set of Dirac brackets is simply from (1) $[6,8,9]$,

$\left[x_{i}, x_{j}\right]_{D}=0, \quad\left[x_{i}, p_{j}\right]_{D}=\left(\delta_{i j}-n_{i} n_{j}\right)$, and

$\left[p_{i}, p_{j}\right]_{D}=-\frac{L_{i j}}{R^{2}}$,

where $L_{i j} \equiv x_{i} p_{j}-x_{j} p_{i}$ is the $i j$-component of the orbital angular momentum. In addition, we have an $S O(N, 1)$ group with generators $p_{i}$ and $L_{i j}$ because we have also [9,34,35],

$\left[L_{i j}, L_{k \ell}\right]_{D}=-\delta_{i \ell} L_{k j}+\delta_{i k} L_{\ell j}+\delta_{j k} L_{i \ell}-\delta_{j \ell} L_{i k}$, and

$\left[L_{i j}, p_{\ell}\right]_{D}=\left(\delta_{i \ell} p_{j}-\delta_{j \ell} p_{i}\right)$.
These relations (12) and (13) hold irrespective of particle being massive or not, relativistic or not. However, in classical mechanics, there is no spin; and these relations (13) are obtained by considering the purely orbital motion. In our approach, we require that these relations (12) and (13) hold true in sense of $[u, v]=i \hbar O\left([u, v]_{D}\right)$. Explicitly, we have,

$$
\begin{aligned}
{\left[p_{i}, p_{j}\right] } & =-i \hbar \frac{J_{i j}}{R^{2}}, \quad\left[J_{i j}, p_{\ell}\right]=i \hbar\left(\delta_{i \ell} p_{j}-\delta_{j \ell} p_{i}\right), \text { and } \\
{\left[J_{i j}, J_{k \ell}\right] } & =i \hbar O\left(\left[J_{i j}, J_{k \ell}\right]_{D}\right) .
\end{aligned}
$$

Here we re-denote $L_{i j}$ by the symbol $J_{i j}$, a symbol denoting generalized angular momentum in quantum mechanics. Our discussion needs a flat space with $N$ cartesian coordinates $x_{i}(i=1,2,3, \ldots, N)$ as the prerequisite. So, for a Dirac fermion on $S^{N-1}$, the FQCs are set up and given by (14), from which the generalized angular momentum $J$ in quantum mechanics is of purely geometric origin, independent of the dynamics.

When quantizing a classical system, we put symmetries on the top priority $[8,9,11,36-38]$. Our philosophy is: The symmetry expressed by the Poisson or Dirac brackets in classical mechanics preserves in quantum mechanics; and so the Hamiltonian is determined by the symmetry. It can be considered a specific demonstration of the fundamental philosophical idea stating that symmetry dictates interactions in quantum mechanics [39]. The philosophy leads us to set out FQCs and DQCs for the non-relativistic and spinless particle, and the most profound consequence is to successfully reproduce of the geometric potential in Hamiltonian and the geometric momentum [11], respectively. In next section, what the DQCs are and how they affect the form of Hamiltonian for a relativistic particle on $\Sigma^{N-1}$ will be formulated.

\section{Dynamical quantum conditions for a relativistic particle on $\Sigma^{N-1}$}

For a relativistic particle whose classical Hamiltonian is $H=\sqrt{(p c)^{2}+\left(\mu c^{2}\right)^{2}}$ with $c$ being the velocity of light and $\mu$ being the mass of the particle, we can obtain two Dirac brackets from (1),

$\left[x_{i}, H\right]_{D}=\frac{p_{i}}{H} c^{2}$, and $\left[p_{i}, H\right]_{D}=-n_{i} \kappa \frac{(c p)^{2}}{H}$,

where $\kappa$ is the first curvature of the geodesic on the hypersurface $\Sigma^{N-1}$ [40]. Notice that Eq. (15) have two important consequences,

$p_{i}=\frac{1}{c^{2}} H\left[x_{i}, H\right]_{D}, \quad$ and $\mathbf{n} \wedge[\mathbf{p}, H]_{D}=0$.

These two relations indicate that in quantum mechanics momentum $\mathbf{p}$ and Hamiltonian $H$ must be compatible with following two quantum conditions, 


$$
\begin{aligned}
& p_{i}=\frac{1}{i \hbar} \frac{H\left[x_{i}, H\right]+\left[x_{i}, H\right] H}{2 c^{2}}, \text { and } \\
& \mathbf{n} \wedge[\mathbf{p}, H]-[\mathbf{p}, H] \wedge \mathbf{n}=0 .
\end{aligned}
$$

These two sets of quantum conditions constitute the socalled DQCs for the relativistic particle on $\Sigma^{N-1}$, which put requirement on the form of Hamiltonian operator.

Three remarks concerning the DQCs are in following.

1. In classical mechanics for a particle, constrained or not, the relativistic velocity $\mathbf{v} \equiv \mathbf{p} c^{2} / H$ (15) can be rewritten as the familiar form, $p=\mu v / \sqrt{1-v^{2} / c^{2}}$. In quantum mechanics, the DQCs imply a definition of the velocity operator $\mathbf{v} \equiv c \alpha=\left(H^{-1} \mathbf{p}+\mathbf{p} H^{-1}\right) c^{2} / 2$ [41] with $\alpha$ are $4 \times 4$ Pauli matrices and in Pauli-Dirac representation we have $\alpha=\left(\begin{array}{ll}0 & \sigma \\ \sigma & 0\end{array}\right)$, while the momentum $\mathbf{p}$ is defined as $\mathbf{p} \equiv(H[\mathbf{x}, H]+[\mathbf{x}, H] H) /\left(2 i \hbar c^{2}\right)$ which is identical to $-i \hbar \nabla$ for motion in flat space. However, it is not the case in quantum mechanics once the motion is constrained. In quantum mechanics, the relativistic Hamiltonian operator $H$ for a particle of any spin in flat space can be easily constructed and it acts on the multicomponent wave functions. However, the construction of such a Hamiltonian for a spin particle on a curved surface or curved space is not an easy task at all. Fortunately, such a Hamiltonian for a Dirac fermion on $S^{2}$ is easily found [16-20,42]. For a Dirac fermion on $S^{2}$, $\mathrm{DQC} \mathbf{n} \wedge[\mathbf{p}, H]-[\mathbf{p}, H] \wedge \mathbf{n}=0$ (17) clearly leads to no presence of geometric potential, as discussed in Sect. 6.

2. For a spinless particle that moves non-relativistically, two Dirac brackets (16) become $[\mathbf{x}, H]_{D} \equiv \mathbf{p} / \mu$ and $\mathbf{n} \wedge$ $[\mathbf{p}, H]_{D}=0$. DQCs take following forms $[8,9,11,38]$,

$$
[\mathbf{x}, H] \equiv i \hbar \frac{\mathbf{p}}{\mu} ; \quad \mathbf{n} \wedge[\mathbf{p}, H]-[\mathbf{p}, H] \wedge \mathbf{n}=0 .
$$

Quantum conditions (2) and (18) constitute the so-called enlarged canonical quantization scheme which gives the unambiguous forms of both the momentum and Hamiltonian $H$ for a non-relativistic free particle [11,38],

$\mathbf{p}=-i \hbar\left(\nabla_{\Sigma}+\frac{M \mathbf{n}}{2}\right) ; \quad H=-\frac{\hbar^{2}}{2 \mu} \nabla_{L B}^{2}+V_{G}$,

where $\nabla_{L B}^{2}=\nabla_{\Sigma} \cdot \nabla_{\Sigma}$ is the usual Laplace-Beltrami operator on the surface $\Sigma^{N-1}$, and $V_{G} \equiv-\hbar^{2}\left(2 K-M^{2}\right)$ $/ 8 \mu$ is the celebrated geometric potential [43-63] in which $K$ is in fact the trace of square of the extrinsic curvature tensor [4], and $\mathbf{p}$ is very geometric momentum without spin connection $[8,9,12]$. Physical consequences resulting from geometric potential and geometric momentum are experimentally confirmed [12,62,63], and more experimentally testable results are under explorations [47-61].

3. In comparison with the overall successes of the DQCs (19) for a non-relativistic and spinless particle on $\Sigma^{N-1}$, we can only say that for a relativistic and spin particle on $\Sigma^{N-1}$ the GCGM and the Hamiltonian must be simultaneously compatible with the DQCs (17). Since the GCGM is already given, we must look for the proper form of the Hamiltonian. It is well-accepted that Hamiltonian for spinless and non-relativistic particle contains the geometric potential, so it is taken for granted that there must be some form of the geometric potential in Hamiltonian for a spin and relativistic particle. Unfortunately, due to the fact that a full understanding of spin connection is still lacking, we can not help but deal with a system on a case-by-case basis. In Sect. 6, we mainly deal with the curvature-induced geometric potential for a Dirac fermion on $S^{2}$. Before it, we give specific FQCs and DQCs in following Sect. 5.

\section{Generalized angular momentum for a Dirac fermion on $S^{2}$}

The surface $S^{2}$ of unit radius can be parameterized by,

$x=\sin \theta \cos \varphi ; \quad y=\sin \theta \sin \varphi ; \quad z=\cos \theta$,

where $\theta$ is the polar angle from the positive $z$-axis with $0 \leq \theta \leq \pi$, and $\varphi$ is the azimuthal angle in the $x y$-plane from the $x$-axis with $0 \leq \varphi<2 \pi$. After some lengthy but straightforward calculations, we can reach a very simple expression for the generally covariant geometric momentum whose three components are given by,

$$
\begin{aligned}
& p_{x}=\Pi_{x}+\sigma_{z} \frac{\hbar}{2} \frac{\cos \theta}{\sin \theta} \sin \varphi, \\
& p_{y}=\Pi_{y}-\sigma_{z} \frac{\hbar}{2} \frac{\cos \theta}{\sin \theta} \cos \varphi, \text { and } p_{z}=\Pi_{z},
\end{aligned}
$$

where $\sigma_{z}=\left(\begin{array}{cc}1 & 0 \\ 0 & -1\end{array}\right)$ is the $z$-component Pauli matrix, and $\Pi_{i}$ are geometric momentum (19) for the particle on $S^{2}[8$, $9,11,38]$,

$$
\begin{aligned}
& \Pi_{x}=-i \hbar\left(\cos \theta \cos \varphi \frac{\partial}{\partial \theta}-\frac{\sin \varphi}{\sin \theta} \frac{\partial}{\partial \varphi}-\sin \theta \cos \varphi\right) ; \\
& \Pi_{y}=-i \hbar\left(\cos \theta \sin \varphi \frac{\partial}{\partial \theta}+\frac{\cos \varphi}{\sin \theta} \frac{\partial}{\partial \varphi}-\sin \theta \sin \varphi\right) ; \\
& \Pi_{z}=i \hbar\left(\sin \theta \frac{\partial}{\partial \theta}+\cos \theta\right) .
\end{aligned}
$$


It has been recognized that spin connection can be interpreted in terms of gauge potential. In GCGM (21), the gauge potential $\mathbf{A}$ is evidently,

$$
\begin{aligned}
\mathbf{A} & =\sigma_{z} \frac{\hbar}{2 r}\left(-\frac{\cos \theta}{\sin \theta} \sin \varphi, \frac{\cos \theta}{\sin \theta} \cos \varphi, 0\right) \\
& =\sigma_{z} \frac{\hbar}{2} \frac{1}{\sqrt{x^{2}+y^{2}+z^{2}}}\left(-\frac{z y}{x^{2}+y^{2}}, \frac{z x}{x^{2}+y^{2}}, 0\right),
\end{aligned}
$$

in which the radius $r=\sqrt{x^{2}+y^{2}+z^{2}}$ is recovered. The magnetic strength $\mathbf{B}$ is,

$\mathbf{B} \equiv \nabla \times \mathbf{A}=-\sigma_{z} \frac{\hbar}{2} \frac{\mathbf{e}_{r}}{r^{2}}$

where $\mathbf{e}_{r} \equiv(x, y, z) / r$. Evidently, the magnetic field is produced by a monopole of unit charge at the center of the sphere $-\delta(r)$, and the eigenvalues $\pm \hbar / 2$ of the Pauli matrix $\sigma_{z} \hbar / 2$ are the effective interaction strength.

The FQCs (14) for a Dirac fermion on $S^{2}$ are explicitly,

$$
\begin{aligned}
{\left[p_{i}, p_{j}\right] } & =-i \hbar \varepsilon_{i j k} \frac{J_{k}}{R^{2}}, \quad\left[J_{i}, p_{j}\right]=i \hbar \varepsilon_{i j k} p_{k}, \text { and } \\
{\left[J_{i}, J_{j}\right] } & =i \hbar \varepsilon_{i j k} J_{k} .
\end{aligned}
$$

These six operators $p_{i}(21)$ and $j_{i}(28)$ constitute all generators of an $S O(3,1)$ group. In consequence, we have following generalized angular momentum,

$J_{x}=L_{x}+\sigma_{z} \frac{\hbar}{2} \frac{\cos \varphi}{\sin \theta}, \quad J_{y}=L_{y}+\sigma_{z} \frac{\hbar}{2} \frac{\sin \varphi}{\sin \theta}$, and $J_{z}=L_{z}$,

where $L_{x}=i \hbar\left(\sin \varphi \partial_{\theta}+\cot \theta \cos \varphi \partial_{\varphi}\right), L_{y}=-i \hbar\left(\cos \varphi \partial_{\theta}\right.$ $\left.-\cot \theta \sin \varphi \partial_{\varphi}\right)$ and $L_{z}=-i \hbar \partial_{\varphi}$ are usually $x, y$ and $z-$ component of the orbital angular momentum, respectively. This generalized angular momentum was first constructed explicitly by Abrikosov [42] who observed the Hamiltonian for massless Dirac fermion to be invariant under a $S U(2)$ group transformation and identified (28) as a consequence. Then, Abrikosov demonstrated it is really generalized angular momentum [42] for the eigenvalues of $J^{2} \equiv J_{i} J_{i}$ are $j(j+1) \hbar^{2}$ with $j=1 / 2,3 / 2,5 / 2, \ldots$ In other words, Abrikosov obtained the generalized angular momentum (28) on the base of dynamics. In contrast, we obtain the same result (28) from both the FQCs (14) and GCGM (6). Moreover, our result (28) applies for particle, massive or massless, relativistic or non-relativistic, irrespective the form of Hamiltonian. In 1993, Ohnuki and Kitakado [34,35] proposed the so-called fundamental algebra for quantum mechanics on $S^{N-1}$ and obtained generators of $S O(N)$ group which reduces, when $N=3$, to be $J_{x}=L_{x}+S y /\left(r^{2}+r z\right)$, $J_{y}=L_{y}-S x /\left(r^{2}+r z\right)$, and $J_{z}=L_{z}$, in which $S$ is a real number rather than an operator in our situation. However, the monopole is the same. Especially, Ohnuki and Kitakado also considered the momentum operators on $S^{N-1}$ but they obtained the geometric one (3) rather than GCGM (6).

\section{No geometric potential for a Dirac fermion on $S^{2}$}

The so-called geometric potential is the additional term in Hamiltonian resulting from quantization. Recently, whether such a curvature-induced geometric potential presents is a topic of considerable controversy [32,64-72], and all use the confining potential formalism but have opposite results. Our approach on the base of the DQCs (17) is totally different from the confining potential formalism, which turns out to be transparent and convincing.

The generally covariant Dirac equation for a fermion on a two-dimensional sphere is [16-20],

$-i \hbar \gamma^{\mu}\left(\partial_{\mu}+\Omega_{\mu}\right) \Psi-\gamma^{0} m \Psi=0$.

where $m \equiv \mu c$ is the reduced mass. The Hamiltonian can be shown to be $[16,42]$,

$H=-i \hbar\left(\sigma_{x}\left(\partial_{\theta}+\frac{1}{2} \frac{\cos \theta}{\sin \theta}\right)+\sigma_{y} \frac{1}{\sin \theta} \partial_{\varphi}\right)+\sigma_{z} m$.

where $\sigma_{x}=\left(\begin{array}{ll}0 & 1 \\ 1 & 0\end{array}\right), \sigma_{y}=\left(\begin{array}{cc}0 & -i \\ i & 0\end{array}\right)$ are, respectively, the $x, y$-component of Pauli matrices. Now, whether a geometric potential exists in the relativistic Hamiltonian (30) is going to be resolved.

First, let us assume the geometric potential in the following most general $2 \times 2$ matrix,

$V_{G}=a_{0} I+a_{x} \sigma_{x}+a_{y} \sigma_{y}+a_{z} \sigma_{z}$

where $\left(a_{0}, a_{x}, a_{y}, a_{z}\right)$ are function of $\theta$ and $\varphi$. The trial Hamiltonian is now $H^{\prime}=H+V_{G}$.

Secondly, we compute three commutators $\left[p_{i}, H^{\prime}\right]$ and the results are, respectively,

$$
\begin{aligned}
{\left[p_{x}, H^{\prime}\right]=} & -\hbar^{2}\left(\sigma_{x} \cos \varphi\left(\sin \theta \partial_{\theta}+\cos \theta\right)\right. \\
& \left.+\sigma_{y}\left(\cos \varphi \partial_{\varphi}-\frac{\sin \varphi}{2}\right)\right)+\left[p_{x}, V_{G}\right] \\
{\left[p_{y}, H^{\prime}\right]=} & -\hbar^{2}\left(\sigma_{x} \sin \varphi\left(\sin \theta \partial_{\theta}+\cos \theta\right)\right. \\
& \left.+\sigma_{y}\left(\sin \varphi \partial_{\varphi}+\frac{\cos \varphi}{2}\right)\right)+\left[p_{y}, V_{G}\right] \\
{\left[p_{z}, H^{\prime}\right]=} & -\hbar^{2}\left(\sigma_{x}\left(\cos \theta \partial_{\theta}+\frac{1}{2 \sin \theta}-\sin \theta\right)\right. \\
& \left.+\sigma_{y} \frac{\cos \theta}{\sin \theta} \partial_{\varphi}\right)+\left[p_{z}, V_{G}\right]
\end{aligned}
$$


Thirdly, since the commutation relations $\left[p_{i}, V_{G}\right]$ in above equation are, respectively,

$$
\begin{aligned}
{\left[p_{x}, V_{G}\right]=} & -i \hbar\left(\cos \theta \cos \varphi \partial_{\theta} V_{G}-\frac{\sin \varphi}{\sin \theta} \partial_{\varphi} V_{G}\right) \\
& +i \hbar \frac{\cos \theta \sin \varphi}{\sin \theta}\left(a_{x} \sigma_{y}-a_{y} \sigma_{x}\right), \\
{\left[p_{y}, V_{G}\right]=} & -i \hbar\left(\cos \theta \sin \varphi \partial_{\theta} V_{G}+\frac{\cos \varphi}{\sin \theta} \partial_{\varphi} V_{G}\right) \\
& -i \hbar \frac{\cos \theta \cos \varphi}{\sin \theta}\left(a_{x} \sigma_{y}-a_{y} \sigma_{x}\right), \\
{\left[p_{z}, V_{G}\right]=} & i \hbar \sin \theta \partial_{\theta} V_{G},
\end{aligned}
$$

we immediately have for $x, y$, and $z$-component of the operator $\mathbf{n} \wedge[\mathbf{p}, H]-[\mathbf{p}, H] \wedge \mathbf{n}$, respectively,

$$
\begin{aligned}
2 i \hbar & \left(\sin \varphi \partial_{\theta} V_{G}+\frac{\cos \theta \cos \varphi}{\sin \theta} \partial_{\varphi} V_{G}\right. \\
& \left.+\frac{\cos ^{2} \theta \cos \varphi}{\sin \theta}\left(a_{x} \sigma_{y}-a_{y} \sigma_{x}\right)\right), \\
2 i \hbar & \left(-\cos \varphi \partial_{\theta} V_{G}+\frac{\cos \theta \sin \varphi}{\sin \theta} \partial_{\varphi} V_{G}\right. \\
& \left.+\frac{\cos ^{2} \theta \sin \varphi}{\sin \theta}\left(a_{x} \sigma_{y}-a_{y} \sigma_{x}\right)\right), \\
& -2 i \hbar\left(\partial_{\varphi} V_{G}+\cos \theta\left(a_{x} \sigma_{y}-a_{y} \sigma_{x}\right)\right) .
\end{aligned}
$$

In final, DQCs $\mathbf{n} \wedge\left[\mathbf{p}, H^{\prime}\right]-\left[\mathbf{p}, H^{\prime}\right] \wedge \mathbf{n}=0$ would be violated unless $\left(a_{0}, a_{x}, a_{y}, a_{z}\right)=(0,0,0,0)$. We see a definite result that there is not geometric potential, i.e., $V_{G}=0$. In other words, Hamiltonian (30) holds true.

An immediate question is whether the geometric potential is possible for a relativistic fermion on all two-dimensional surfaces $\Sigma^{2}$, i.e., whether the conclusion above holds true in general. At present, we are still struggling for the answer to this question. However, explicit calculations on the case-bycase basis show that the fermion experiences no geometric potential on surfaces such as torus, minimal surface, etc., which will be soon published elsewhere.

\section{Conclusions}

For a particle that is constrained on an $(N-1)$-dimensional curved surface, the geometric momentum (3) has stood both theoretical examinations and experimental testifications. To make it generally covariant so as to be applicable to the spin particles, a simple replacement suffices through ordinary derivative $\partial_{\mu}$ in gradient operator $\nabla_{\Sigma} \equiv \mathbf{e}^{\mu} \partial_{\mu}$ by its generally covariant derivative $\partial_{\mu} \longrightarrow \partial_{\mu}+i \Omega_{\mu}$. The generally covariant geometric momentum is thus resulted in, and a remarkable consequence of it is the manifestation of the gauge potential $A_{\mu} \equiv-\hbar \Omega_{\mu}$.
A general formalism when quantizing a classical system is established, in which we have both FQCs and DQCs. The FQCs for a Dirac fermion on $S^{N-1}$ lead us to a generalized angular momentum, while the DQCs for a Dirac fermion on $\Sigma^{N-1}$ offer us a way to check whether the geometric potential presents for relativistic spin particle on any hypersurface $\Sigma^{N-1}$. In present paper, we obtain the generalized angular momentum for $S^{2}$, which is demonstrated to be of geometric origin but was reported before on the dynamical consideration. Moreover we show that for a Dirac fermion on $S^{2}$, no geometric potential is permissible.

Acknowledgements This work is financially supported by National Natural Science Foundation of China under Grant No. 11675051.

Data Availability Statement This manuscript has no associated data or the data will not be deposited. [Authors' comment: All theoretical derivations can be easily reproduced. In case of needed, please send request to the corresponding author.]

Open Access This article is distributed under the terms of the Creative Commons Attribution 4.0 International License (http://creativecomm ons.org/licenses/by/4.0/), which permits unrestricted use, distribution, and reproduction in any medium, provided you give appropriate credit to the original author(s) and the source, provide a link to the Creative Commons license, and indicate if changes were made.

Funded by $\mathrm{SCOAP}^{3}$.

\section{References}

1. P.A.M. Dirac, The Principles of Quantum Mechanics, 4th edn. (Oxford Univ, Oxford, 1967), pp. 87-112-114

2. P.A.M. Dirac, Lectures on Quantum Mechanics (Yeshiva University, New York, 1964)

3. T. Homma, T. Inamoto, T. Miyazaki, Schrödinger equation for the nonrelativistic particle constrained on a hypersurface in a curved space. Phys. Rev. D 42, 2049 (1990)

4. M. Ikegami, Y. Nagaoka, S. Takagi, T. Tanzawa, Quantum mechanics of a particle on a curved surface: comparison of three different approaches. Prog. Theor. Phys. 88, 229-249 (1992)

5. J.R. Klauder, S.V. Shabanov, Coordinate-free quantization of second-class constraints. Nucl. Phys. B 511, 713 (1998)

6. S. Weinberg, Lectures on Quantum Mechanics, 2nd edn. (Cambridge University Press, Cambridge, 2015), pp. 335-340

7. Q.H. Liu, C.L. Tong, M.M. Lai, Constraint-induced mean curvature dependence of Cartesian momentum operators. J. Phys. A 40, 4161-4168 (2007)

8. Q.H. Liu, L.H. Tang, D.M. Xun, Geometric momentum: the proper momentum for a free particle on a two-dimensional sphere. Phys. Rev. A 84, 042101 (2011)

9. Q.H. Liu, Geometric momentum for a particle constrained on a curved hypersurface. J. Math. Phys. 54, 122113 (2013)

10. Q.H. Liu, Geometric momentum and a probe of embedding effects. J. Phys. Soc. Japan 82, 104002 (2013)

11. D.K. Lian, L.D. Hu, Q.H. Liu, Geometric potential and Dirac quantization. Ann. Phys. (Berlin) 530, 1700415 (2018)

12. R. Spittel, P. Uebel, H. Bartelt, M.A. Schmidt, Curvature-induced geometric momenta: the origin of waveguide dispersion of surface plasmons on metallic wires. Opt. Express 23, 12174-12188 (2015)

13. Y.L. Wang, H. Jiang, H.S. Zong, Geometric influences of a particle confined to a curved surface embedded in three-dimensional Euclidean space. Phys. Rev. A 96, 022116 (2017) 
14. A.V. Golovnev, Int. J. Geom. Methods Mod. Phys. 3, 655 (2006)

15. D.R. Brill, J.A. Wheeler, Interaction of neutrinos and gravitational fields. Rev. Mod. Phys. 29, 465-479 (1957)

16. M.A.H. Vozmediano, M.I. Katsnelson, F. Guinea, Gauge fields in grapheme. Phys. Rep. 496, 109-148 (2010)

17. D.-H. Lee, Surface states of topological insulators: the Dirac fermion in curved two-dimensional spaces. Phys. Rev. Lett. 103, 196804 (2009)

18. A. Iorio, G. Lambiase, The Hawking-Unruh phenomenon on grapheme. Phys. Lett. B 716, 334-337 (2012)

19. A. Iorio, G. Lambiase, Quantum field theory in curved graphene spacetimes, Lobachevsky geometry, Weyl symmetry, Hawking effect, and all that. Phys. Rev. D 90, 025006 (2014)

20. A. Iorio, Curved spacetimes and curved graphene: a status report of the Weyl symmetry approach. Int. J. Mod. Phys. D 24, 1530013 (2015)

21. N. Ogawa, Remark on the classical solution of the Chaplygin gas as d-branes. Mod. Phys. Lett. A 12, 1583-1588 (1997)

22. K. Fujii, N. Ogawa, S. Uchiyama, N.M. Chepilko, Geometrically induced gauge structure on manifolds embedded in a higherdimensional space. Int. J. Mod. Phys. A 12, 5235-5277 (1997)

23. K. Akama, Unification scenario of the gravitational and gauge forces via the higher dimensional soliton. Prog. Theor. Phys. 78, 184-188 (1987)

24. P. Maraner, C. Destri, geometry-induced Yang-Mills fields in constrained quantum mechanics. Mod. Phys. Lett. A 8, 861-868 (1993)

25. K. Fujii, N. Ogawa, Generalization of geometry-induced gauge structure to any dimensional manifold. Prog. Theor. Phys. 89, 575578 (1993)

26. P. Maraner, A complete perturbative expansion for quantum mechanics with constraints. J. Phys. A Math. Gen. 28, 2939-2951 (1995)

27. P. Maraner, Monopole gauge fields and quantum potentials induced by the geometry in simple dynamical systems. Ann. Phys. 246, 325-346 (1996)

28. N.M. Chepilko, K. Fujii, Gauge and supersymmetric structure of quantum mechanics on hypersurfaces embedded in Euclidean space. Phys. Atom. Nucl. 58, 1063-1071 (1995)

29. H. Grundling, C.A. Hurst, Constrained dynamics for quantum mechanics. I. Restricting a particle to a surface. J. Math. Phys. 39, 3091-3119 (1998)

30. K. Akama, T. Hattori, Induced field theory on the Brane worldgravity, extrinsic curvature, and gauge fields. Mod. Phys. Lett. A 15, 2017-2026 (2000)

31. P.C. Schuster, R.L. Jaffe, Quantum mechanics on manifolds embedded in Euclidean space. Ann. Phys. 307, 132-143 (2003)

32. P. Maraner, J.K. Pachos, Universal features of dimensional reduction schemes from general covariance breaking. Ann. Phys. 323, 2044-2072 (2008)

33. Y. Ohnuki, S. Kitakado, On quantum mechanics on compact space. Mod. Phys. Lett. A 7, 2477-2482 (1992)

34. Y. Ohnuki, S. Kitakado, Fundamental algebra for quantum mechanics on $S^{D}$ and gauge potentials. J. Math. Phys. 34, 2827 (1993)

35. M. Hirayama, H.M. Zhang, T. Hamada, Induced gauge structure of quantum mechanics on $S^{D}$. Prog. Theor. Phys. 97, 679-689 (1997)

36. D.M. Xun, Q.H. Liu, X.M. Zhu, Quantum motion on a torus as a submanifold problem in a generalized Dirac's theory of secondclass constraints. Ann. Phys. (NY) 338, 123-133 (2013)

37. D.M. Xun, Q.H. Liu, Geometric momentum in the Monge parametrization of two dimensional sphere. Ann. Phys. (NY) 341, 132-141 (2014)

38. Z.S. Zhang, S.F. Xiao, D.M. Xun, Q.H. Liu, An enlarged canonical quantization scheme and quantization of a free particle on twodimensional sphere. Commun. Theor. Phys. 63, 19-24 (2015)

39. C.N. Yang, Einstein's impact on theoretical physics. Phys. Today 33, 42-49 (1980)
40. L.D. Hu, D.K. Lian, Q.H. Liu, The centripetal force law and the equation of motion for a particle on a curved hypersurface. Eur. Phys. J. C 76, 655 (2016)

41. K.Y. Bliokh, Mark R. Dennis, F. Nori, Position, spin, and orbital angular momentum of a relativistic electron. Phys. Rev. A 96, 023622 (2017)

42. A.A. Abrikosov, Fermion states on the sphere $S^{2}$. Int. J. Mod. Phys. A 17, 885-889 (2002)

43. H. Jensen, H. Koppe, Quantum mechanics with constraints. Ann. Phys. 63, 586-591 (1971)

44. R.C.T. da Costa, Quantum mechanics of a constrained particle. Phys. Rev. A 23, 1982-1987 (1981)

45. R.C.T. da Costa, Constraints in quantum mechanics. Phys. Rev. A 25, 2893-2900 (1982)

46. G. Ferrari, G. Cuoghi, Schrődinger equation for a particle on a curved surface in an electric and magnetic field. Phys. Rev. Lett. 100, 230403 (2008)

47. M.V. Entin, L.I. Magarill, Spin-orbit interaction of electrons on a curved surface. Phys. Rev. B 64, 085330 (2001)

48. A.V. Chaplik, R.H. Blick, On geometric potentials in quantumelectromechanical circuits. New J. Phys. 6, 33 (2004)

49. V. Atanasov, R. Dandoloff, A. Saxena, Geometry-induced charge separation on a helicoidal ribbon. Phys. Rev. B 79, 033404 (2009)

50. Y.N. Joglekar, A. Saxena, Curvature-induced $\mathrm{p}-\mathrm{n}$ junctions in bent or folded bilayer graphene sheets. Phys. Rev. B 80, 153405 (2009)

51. S. Ono, H. Shima, Tuning the electrical resistivity of semiconductor thin films by nanoscale corrugation. Phys. Rev. B 79, 235407 (2009)

52. H. Shima, H. Yoshioka, J. Onoe, Geometry-driven shift in the Tomonaga-Luttinger exponent of deformed cylinders. Phys. Rev. B 79, 201401(R) (2009)

53. V. Atanasov, A. Saxena, Tuning the electronic properties of corrugated graphene: confinement, curvature, and band-gap opening. Phys. Rev. B 81, 205409 (2010)

54. S. Ono, H. Shima, Low-temperature resistivity anomalies in periodic curved surfaces. Phys. E Low Dimens. Syst. Nanostruct. 42, 1224-1227 (2010)

55. F.T. Brandt, J.A. Sáchez-Monroy, Induced magnetic moment for a spinless charged particle in the thin-layer approach. Europhys. Lett. 111, 67004 (2015)

56. C. Ortix, J. van den Brink, Absence of anomalous couplings in the quantum theory of constrained electrically charged particles. Phys. Rev. B 83, 113406 (2011)

57. D. Schmeltzer, Dirac's method for constraints: an application to quantum wires. J. Phys. Condens. Matter 23, 155601 (2011)

58. E.O. Silva, S.C. Ulhoa, F.M. Andrade, C. Filgueiras, R.G.G. Amorim, Quantum motion of a point particle in the presence of the Aharonov-Bohm potential in curved space. Ann. Phys. (N.Y.) 362, 739-751 (2015)

59. C. Filgueiras, E.O. Silva, 2 DEG on a cylindrical shell with a screw dislocation. Phys. Lett. A 379, 2110-2115 (2015)

60. Y.L. Wang, L. Du, C.T. Xu, X.J. Liu, H.S. Zong, Pauli equation for a charged spin particle on a curved surface in an electric and magnetic field. Phys. Rev. A 90, 042117 (2014)

61. Y.L. Wang, M.Y. Lai, F. Wang, H.S. Zong, Y.F. Chen, Geometric effects resulting from square and circular confinements for a particle constrained to a space curve. Phys. Rev. A 97, 042108 (2018)

62. A. Szameit, F. Dreisow, M. Heinrich, R. Keil, S. Nolte, A. Tünnermann, S. Longhi, Geometric potential and transport in photonic topological crystals. Phys. Rev. Lett. 104, 150403 (2010)

63. J. Onoe, T. Ito, H. Shima, H. Yoshioka, S. Kimura, Observation of Riemannian geometric effects on electronic states. Europhys. Lett. 98, 27001 (2012)

64. S. Matsutani, H. Tsuru, Physical relation between quantum mechanics and solitons on a thin elastic rod. Phys. Rev. A 46, 1144-1147 (1992) 
65. M. Burgess, B. Jensen, Fermions near two-dimensional surfaces. Phys. Rev. A 48, 1861 (1993)

66. S. Matsutani, The relation between the modified Korteweg-de Vries equation and an anomaly of the Dirac field on a thin elastic rod. Prog. Theor. Phys. 91, 1005-1037 (1994)

67. S. Matsutani, A constant mean curvature surface and the Dirac operator. J. Phys. A Math. Gen. 30, 4019 (1997)

68. S. Matsutani, Dirac operator of a conformal surface immersed in R4: further generalized Weierstrass relation. Rev. Math. Phys. 12, $431(2000)$

69. V. Atanasov, A. Saxena, Electronic properties of corrugated graphene: the Heisenberg principle and wormhole geometry in the solid state. J. Phys. Condens. Matter 23, 175301 (2011)
70. M.A. Olpak, Dirac equation on a curved $(2+1)$ dimensional hypersurface. Mod. Phys. Lett. A 27, 250016 (2012)

71. V. Atanasov, A. Saxena, Helicoidal graphene nanoribbons: chiraltronics. Phys. Rev. B 92, 035440 (2015)

72. F.T. Brandt, J.A. Sáchez-Monroy, Dirac equation on a curved surface. Phys. Lett. A 380, 3036-3043 (2016) 